\title{
The Value of Ultrasound in the Diagnosis of Children's Appendicitis
}

\author{
Yan Liu, Ruizhe Hou, Ruizhi Hou* \\ China-Japan Union Hospital of Jilin University, Changchun, Jilin, 130021, China \\ *Corresponding author
}

Keywords: ultrasound diagnosis, children appendicitis, application value, reference basis

\begin{abstract}
The paper explored the effect of ultrasound in the diagnosis of appendicitis in children, and analyzed its clinical application value, so as to provide some references for clinical diagnosis. From January, 2015 to December, 2017, 196 cases of children with appendicitis were selected as the study objects. According to the clinical data statistics ultrasonic diagnosis, the authors analyzed the application value of ultrasound in the diagnosis of children appendicitis to provide some references for clinical practice. By comparison with the results of pathological examination, 180 cases were matched with the type of appendicitis, and 91.84 percent were diagnosed correctly. Moreover, 16 cases were missed diagnosis, and the missed diagnosis rate was 8.16 percent. Among them, 8 cases were misdiagnosed as intestinal dilatation, and 8 cases were misdiagnosed as mesenteric lymphadenopathy. After re-exploration, it was proved to be simple and suppurative appendicitis. The high frequency color Doppler ultrasound was proved to have a high diagnostic coincidence rate, simple operation and low diagnostic cost in the diagnosis of acute appendicitis in children. It was worth popularizing and applying in the clinic.
\end{abstract}

\section{Introduction}

Appendicitis is a common type of acute abdomen. Children with appendicitis are more than five years old. The incidence of appendicitis in children was significantly lower than that in adults. But the children's condition is more serious, and the disease progress is faster with higher mortality. We should strengthen the early diagnosis and typing of appendicitis in children. According to the actual condition of children, targeted treatment is the focus of clinical treatment of children with appendicitis. Ultrasonic examination is a common method in clinical diagnosis of adult appendicitis, but the clinical manifestations and signs of appendicitis in children are different from those in adult appendicitis, and the diagnosis is more difficult. In order to determine the effect of ultrasound diagnosis, 196 children with appendicitis admitted from January, 2015 to December, 2017 were diagnosed by ultrasound. The following is a retrospective summary of the inspection process.

\section{Data and Methods}

\subsection{Clinical data}

A total of 196 children with appendicitis were selected from January, 2015 to December, 2017, including 102 male and 94 female. The average age is $6.2 \pm 2$. 3 years and the age is between 5 to 14 years old. All subjects were excluded from severe immune diseases, blood disorders and mental disorders, so we can ensure that no adverse effects on the results were observed.

\subsection{Methods}

With PHILIPS ELIPE ultrasonic diagnostic instrument, the patient was supine, and the whole abdomen was scanned with a convex array probe. First, the appendicitis was examined by the right ventral longitudinal and transverse resection of the ascending colon cecum and the right lower abdomen by random scanning of the right lower ventral Mackney's point with the ileocecal flap moving along the clockwise direction. We focused on the most obvious part of the patient's right lower abdomen tenderness, carefully looking for the appendix, observing the shape, boundary, echo 
of the appendix. Moreover, we should observe the thickening and edema of the intestinal wall around the appendix, the accumulation of fluid around the appendix, the lymph node enlargement around the root of the appendix, and record the results of ultrasound examination, and then repeat the above scanning process with the linear array probe. The results of ultrasonic examination were recorded.

\subsection{Observation indicators}

The ultrasonic diagnosis of 196 children with appendicitis was counted and compared with operation and pathology. The accuracy of ultrasonic diagnosis was calculated and the value of ultrasound in the diagnosis of infantile appendicitis was analyzed.

\section{Results}

\subsection{Comparison between ultrasound diagnosis and surgical pathology}

In this paper, 196 children with acute appendicitis were examined by high frequency color Doppler ultrasound and compared with the results of operation and pathology. There were 180 cases of appendicitis, and the diagnostic coincidence rate was 91.84 and the misdiagnosis rate was 16 cases; the false missed diagnosis rate was 8.16. The detailed statistical data are shown in Table 1.

Table 1 Comparison of Ultrasound Diagnosis with Surgical Pathology

\begin{tabular}{cccc}
\hline Appendicitis Type & $\begin{array}{c}\text { Diagnostic } \\
\text { Coincidence (case) }\end{array}$ & $\begin{array}{c}\text { Misdiagnosis } \\
\text { (case) }\end{array}$ & $\begin{array}{c}\text { Diagnostic coincidence rate } \\
(\%)\end{array}$ \\
\hline simple acute appendicitis, $(\mathrm{n}=62)$ & 58 & 4 & 93.55 \\
Gangrenous Appendicitis $(\mathrm{n}=30)$ & 26 & 4 & 86.67 \\
Purulent Appendicitis(n=96) & 88 & 8 & 91.67 \\
Periappendiceal Abscess $(\mathrm{n}=8)$ & 8 & 0 & 100 \\
Total Amount (n=196) & 180 & 16 & 91.84 \\
\hline
\end{tabular}

\subsection{Ultrasonic imaging features of various types of appendicitis}

First, simple appendicitis: Ultrasonography showed slight enlargement of the appendix (external diameter) smm. In the image, the contour of the appendix was clearly displayed, and the mucous membrane of the appendix wall was rough; the layer was clear, and a small amount of liquid anechoic could be seen in the cavity. Blood flow imaging shows abundant blood flow signal in the appendiceal wall. Second, gangrenous appendicitis: Ultrasound images showed slight or obvious enlargement of the appendix, and the contour of the appendix and the layer of the wall of the appendix were obviously blurred, especially the mucous membrane could only show partial or completely unclear. A large amount of liquid anechoic can be found in the appendix, or a small amount of effusion is found and the echo is weak. Flow imaging shows little or no blood flow signal in the appendiceal wall. Third, suppurative appendicitis: Ultrasonic images show that the appendix is obviously enlarged with an external diameter of 8 to $16 \mathrm{~mm}$ and the appendiceal wall was unevenly thickened and stratified $(4-7 \mathrm{~mm})$. In the appendix cavity, irregular liquid dark area was observed, and a large, punctate echo was observed. Display of stripe blood flow signal in appendiceal tissue by blood flow imaging. Fourth, periappendiceal abscess: Ultrasonic images show that the appendiceal structure is rather cluttered, so it is unable to clearly display the appendiceal structure with blurred boundaries and irregular shape. Uneven masses could be seen in the lesion area, irregular flaky fluid dark areas appeared in the appendix cavity, and spot spots and flaky strong echoes appeared. Around the mass, the pelvic cavity appeared irregular anechoic, and the appendiceal cavity sound transmission effect was poor.

\subsection{Analysis of misdiagnosed cases}

In the diagnosis of high frequency color Doppler ultrasound, 16 cases were misdiagnosed, of which 8 cases were intestinal dilatation and 8 cases were mesenteric lymphadenopathy. Because the early symptoms of children have no obvious specificity, the main features are as follows: First, due 
to more gas in the lower abdomen and tension in the abdominal muscles of the children, the intestinal tube in the abdomen can be seen to dilate obviously when the probe is pressurized, which leads to the inability to accurately explore the relationship between the appendix and its surroundings. Second, there was no obvious swelling in ileocecal part of appendicitis, lymph node enlargement and no inflammatory sign of appendicitis were found in the right lower abdomen of children with tenderness, so ultrasound images showed that there was no obvious swelling in ileocecal part of appendix. During the period of hospital observation, the symptoms of lower abdominal pain in 16 cases were obviously aggravated. High frequency color Doppler ultrasonography showed that 8 cases were simple appendicitis and 8 cases were suppurative appendicitis. The diagnostic results of the patients were in good agreement with the operation and pathology.

\section{Discussions}

Acute appendicitis is a common acute abdomen in children. Because the dissociation of the ileocecal part of children is higher than that of adults, the position of appendicitis is different, and its change range can be down to the pelvic cavity, the upper abdomen and even the lower margin of liver. In the early stage of acute appendicitis in children, abdominal pain, abdominal muscle tension and local signs are often not obvious. In addition, most of the children can not accurately describe the location of pain, resulting in accurate diagnosis having a certain degree of difficulty. As the disease is more common in preschool infants and children, the development of the greater omentum is not perfect and other factors, leading to the spread of inflammatory reaction and aggravated systemic symptoms. Even a short period of time, severe conditions such as diffuse peritonitis caused by perforation of the appendix.

Ultrasound is a common imaging method for clinical diagnosis of diseases. Ultrasound image has high resolution and clear imaging. It can not only understand the shape of appendicitis, but also the echo of appendix and surrounding tissue state, so that to assist the diagnosis of diseases effectively.

The diagnosis has the characteristics of non-invasive and non-radiation, and the diagnostic safety is high. Ultrasonic diagnosis can clearly display the shape and size of the appendix and abscess in the peritoneal cavity of the mesenteric membrane. At the same time, the internal blood flow can be grasped by AXSI, and the focus can be displayed more accurately and intuitively. This can avoid repeated reactionary blind exploration, and is easy to operate so that to ease the patient's pain. The ultrasonic diagnostic criteria for acute appendicitis in children were enlargement of appendix with diameter of appendix exceeding $6 \mathrm{~mm}$. Appendectomy and appendiceal effusion is helpful for the diagnosis of appendectomy. It also showed no peristalsis, rough mucosa, interrupted echo, poor smooth serosa and enhanced echo. When appendicitis was enlarged, the peritonitis appeared accompanied by a small amount of effusion between adjacent intestines, suggesting suppurative appendicitis. AXSI showed enhanced color blood flow signal in the appendiceal region during inflammatory hyperemia in periappendiceal tissue. The disease should be distinguished from intussusception and acute mesenteric lymphadenitis. But acute mesenteric lymphadenitis is simple enlargement of mesenteric lymph nodes with few other signs.

Acute simple appendicitis was the early change of the lesion, and the appendicitis was slightly swollen; there was a small amount of exudation in the cavity, and the display rate of direct and indirect signs was relatively low, and the ultrasonic examination was easy to miss diagnosis. When the lesion develops into suppurative or gangrenous appendicitis, the appendix is hyperemia and edema, and blood pus in the cavity, and bloody or purulent fluid accumulation in the abdominal cavity. At this time, the detection rate of direct and indirect signs was significantly increased, and the diagnostic accuracy of ultrasound was improved significantly. When the appendiceal gangrene, perforation is wrapped by the greater omentum to form periappendiceal abscess, the sonographic appearance is mixed mass, and the specificity is high; moreover, the diagnostic accuracy of ultrasound can be up to 100 percent. When the clinical symptoms of some patients are consistent with acute appendicitis, and ultrasound can not show direct signs, we can use the indirect signs 
observed by ultrasound to indicate the existence of acute appendicitis. This is mainly because patients with acute appendicitis are often accompanied by these indirect signs.

In order to verify the value of ultrasound in children with appendicitis, 196 children with appendicitis were selected from January, 2015 to December, 2017. According to clinical data, ultrasonic diagnosis was statistically analyzed. According to the results of operation and pathology, 180 cases of appendicitis were found to be consistent, and the diagnostic coincidence rate was 91.84; while, the misdiagnosis rate was 16 cases and the missed diagnosis rate was 8.16. 8 cases were misdiagnosed as intestinal dilatation and 8 cases were misdiagnosed as mesenteric lymphadenopathy, which was proved to be simple and suppurative appendicitis by re-exploration. This indicates that the high frequency color Doppler ultrasound technique has higher diagnostic accuracy in the diagnosis of acute appendicitis in children with simple operation and low diagnosis cost, which is worthy of clinical application.

To sum up, the application of ultrasound in the diagnosis of appendicitis in children has a good effect, and it can effectively assist the diagnosis and differential diagnosis of diseases in children with appendicitis. It has high application value and can provide some reference for clinical diagnosis.

\section{References}

[1] Li Hongying. Clinical Value of High Frequency Color Doppler Ultrasound in the Diagnosis of Acute Appendicitis in Children [J]. Heilongjiang Medicine, 2014, 27(03): 687-689.

[2] Chen Qiuyan, Zhang Jianfei, Zhang Qiaoyi, Luo Jianhua. The Application of Ultrasonography in the Clinical Diagnosis of Appendicitis in Children [J]. China People's Medicine, 2014, 26(16): 54-56.

[3] Li Yan, Wu Mengqi. Discussion on the Value of Ultrasonic Examination in the Diagnosis and Classification of Children's Appendicitis [J]. Chinese and Foreign Medical Research, 2014, 12(23): 54-55.

[4] Sun Jianshe, Gao Guoqiang, Wang Rumei, Zhang Jingru. The Value of Ultrasound in the Diagnosis of Children's Appendicitis [J]. Chinese Practical Medicine, 2016, 11(15): 70-71.

[5] Ge Yurong, Zhou Yan. Diagnostic Value of Color Doppler Ultrasound in 60 Children with Appendicitis [J]. Guizhou Medicine, 2016, 40(07): 773-774.

[6] Wang Lin. Clinical Application of Abdominal Ultrasound in the Diagnosis of Acute Appendicitis in Children [J]. Health for All (Academic Edition), 2015, 9(03): 9-10. 